\title{
UK Renal Registry 14th Annual Report: Appendix D Methodology used for Analyses of PCT/HB Incidence and Prevalence Rates and of Standardised Ratios
}

Described here are the methods for calculating the standardised incidence ratios for the incident UK RRT cohort, the standardised prevalence ratios for the total UK RRT cohort and the ratios for prevalent transplant patients.

\section{Patients}

For the incidence rate analyses, all new cases recorded by the UK Renal Registry (UKRR) as starting RRT in each year were included. For the prevalence rates analyses, prevalent patients at the end of the year were included. The analyses used the patient postcode rather than the GP postcode. Each postcode was matched to a 2001 Census output area and hence to the relevant area code.

\section{Years used}

Analyses have been completed for each of the last 6 years. Combined analyses have also been done using the data from as many of the years as are available for each area. This combined analysis is useful for the incidence rates and rate ratios analyses as there can be small numbers of incident patients particularly in the smaller areas.
C) 2012 S. Karger AG, Basel

$1160-2110 / 12 / 1185-0279 \$ 38.00 / 0$

Accessible online at: www.karger.com/nec

\section{Geography}

The areas used were the 146 English primary care trusts (PCTs), the five English care trusts, the seven Welsh Local Health Boards, the fourteen Scottish Health Boards and the five Health and Social Care Trusts in Northern Ireland - these different types of area are collectively called PCT/HBs here. These areas in England are likely to undergo significant reorganisation in the next few years with the introduction of clinical commissioning groups. There will be more of these areas each containing smaller population groups, although the boundaries have yet to be finalised.

Areas included in the UK Registry 'covered' population

This year all renal centres are again sending data to the Registry so coverage of the UK is complete for 2008 to 2010. In previous years, not all renal centres were sending data to the UKRR. This meant that estimates could not be obtained for all PCT/HBs but only for those which were covered by the Registry in the relevant year. The UKRR identified all areas which were estimated to have complete coverage and analyses were restricted to those areas. Whether an area was covered or not was dependant on whether the renal centre in the area was sending data to the UKRR and whether there were any overlapping areas with renal centres not yet connected to the UKRR.

UK Renal Registry, Southmead Hospital, Southmead Road, Bristol, BS10 5NB, UK

Email: renalregistry@renalregistry.nhs.uk 


\section{Population data}

Mid-2010 population estimates were obtained from the Office for National Statistics (ONS) website (www.statistics.gov.uk) by $\mathrm{PCT} / \mathrm{HB}$, gender and age group (for Northern Ireland the ONS population data were aggregated at the Registry from district council to Health and Social Care Trust level). These 2010 estimates have been extrapolated by the ONS from the 2001 census data. The areas range in population size from 20,000 (Orkney) to 1.3 million (Hampshire).

This 2010 population data is used for the analysis for each year. As the analyses only cover six years this was a reasonable approximation.

\section{Calculation of rates and rate ratios}

\section{Crude rates}

The crude rates, per million population ( $\mathrm{pmp}$ ), were calculated for each PCT/HB for each year:

$$
1,000,000 \times(\text { observed number }) /(\text { population size })
$$

For the combined years analyses the observed cases are summed over the available years and the population is multiplied by the number of years that the area has been covered. For example, if area $\times$ (population 100,000) became an area covered for the first time in 2008 and had 14 new patients in 2008, 10 in 2009 and 19 in 2010 then the combined years crude acceptance rate would be $1,000,000 \times(14+10+19) /(3 \times 100,000)=143 \mathrm{pmp}$. Again, this is a rate per million population per year. It is an average over the available years.

Confidence intervals have not been calculated for these (single or combined years) rates but, if required, an assessment can be made of whether the rate for a given area is consistent with the rate in the whole covered population. This can be done by using the figures provided here showing the confidence intervals around the overall average rates for a range of $\mathrm{PCT} / \mathrm{HB}$ population sizes. These are figures D.1 and D.2 for incidence rates, and D.3 and D.4 for prevalence rates.

Note that when using the confidence interval figures to assess how different an area's combined years crude rate is from the overall average, the population shown on the $\mathrm{x}$-axis should be the area's population multiplied by the number of years of data that has been used (e.g. 3 for the example above). By doing this, the confidence

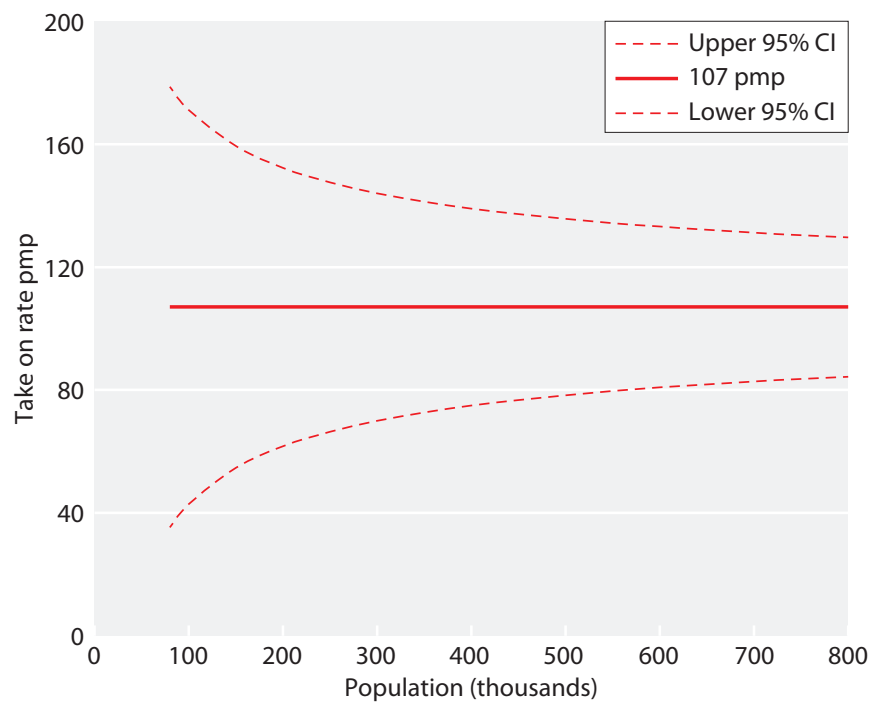

Fig. D.1. $95 \%$ confidence limits for take on rate of $107 \mathrm{pmp}$ for population size $80,000-800,000$

intervals obtained become narrower as the analysis is now based on more than one year of data.

These confidence intervals have been obtained using the Normal approximation to the Poisson distribution. For the incident analyses, confidence intervals have only been calculated around the overall average for populations of over 80,000 . This is because below this level the number of cases you would expect per area is low and so the Poisson distribution is skewed and

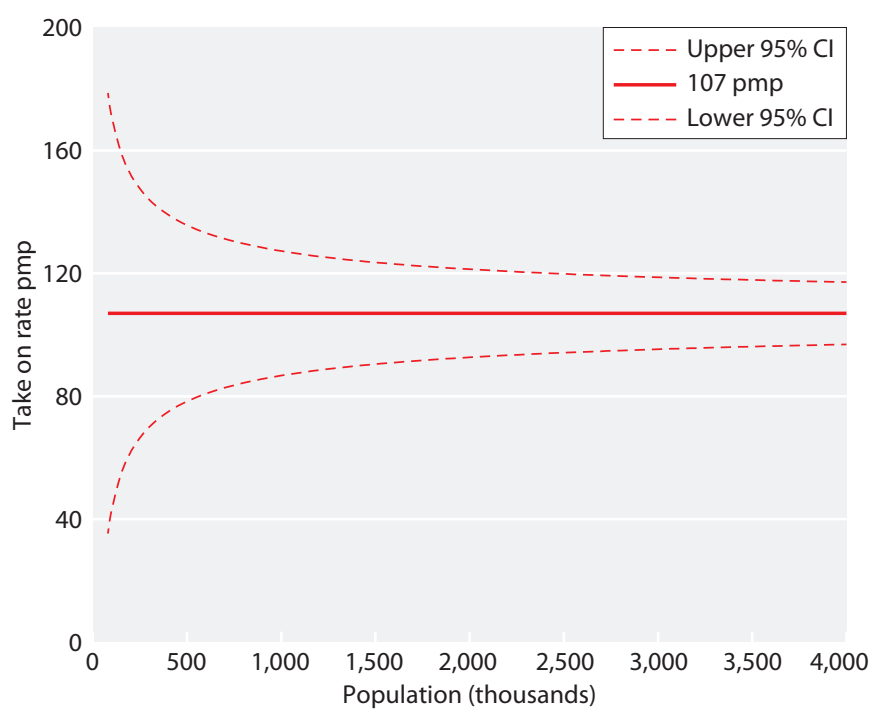

Fig. D.2. $95 \%$ confidence limits for take on rate of $107 \mathrm{pmp}$ for population size $80,000-4$ million 


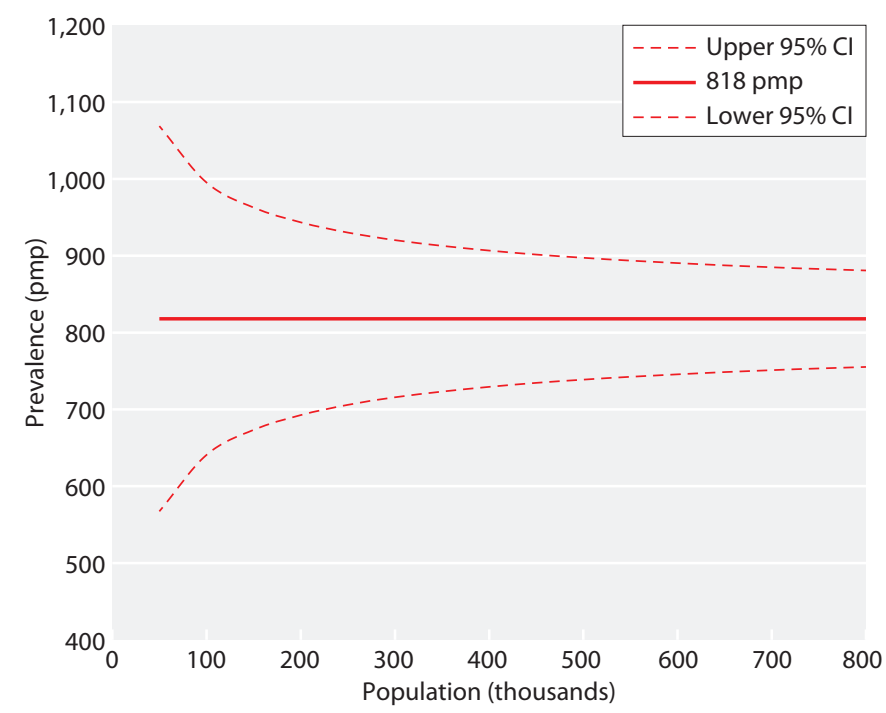

Fig. D.3. $95 \%$ confidence limits for prevalence of $818 \mathrm{pmp}$ for catchment population size 50,000-600,000

the Normal approximation is not appropriate. Due to prevalence rates being higher, confidence intervals can be obtained using this method for lower population sizes.

\section{Standardised incidence/prevalence ratios (SIR/SPR or $S R$ )}

There are large differences in incidence and prevalence rates for RRT between age and gender groups. As there are also differences in the age/gender breakdowns of

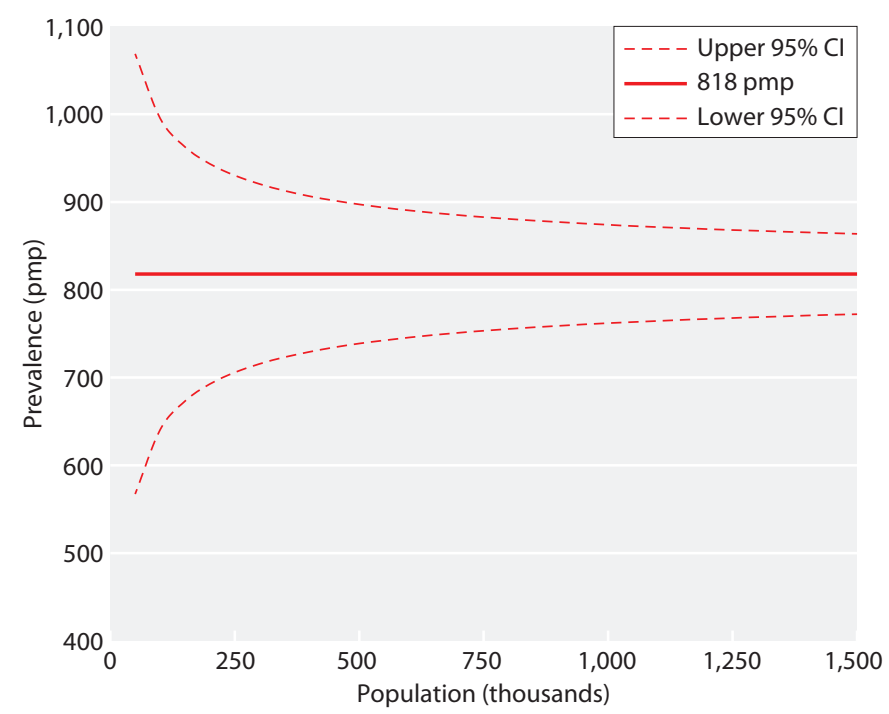

Fig. D.4. $95 \%$ confidence limits for prevalence of $818 \mathrm{pmp}$ for catchment population size 50,000-1.5 million the different areas it is useful to produce estimates standardised for age and gender. The method used is indirect standardisation.

Observed cases $\left(\mathrm{O}_{\mathrm{i}}\right)$ were calculated by summing all cases in all age and gender bands for each PCT/HB. Expected cases $\left(\mathrm{E}_{\mathrm{i}}\right)$ for each PCT/HB were calculated as follows:

Overall crude rates (for each year) were calculated for the whole covered population (the standard population) by summing the observed numbers, over the PCT/HBs, for each age/gender band and dividing this by the total covered population in that age/ gender band. These crude rates (by age/gender band) were then multiplied by the population each $\mathrm{PCT} / \mathrm{HB}$ has in each band to give the number of cases expected in that band if that PCT/HB had the same rates as the standard population.

These expected numbers were then summed over the age/gender bands to give an expected total number of cases in each $\mathrm{PCT} / \mathrm{HB}$. The age/gender standardised ratio for PCT/HB i is then $\mathrm{O}_{\mathrm{i}} / \mathrm{E}_{\mathrm{i}}$.

The expected number of cases is the number you would see if the rates seen in the standard population applied to that individual PCT/HB's age/gender breakdown. 95\% confidence intervals were calculated for each area using an error factor (EF) as follows:

$$
\begin{aligned}
\mathrm{LCL} & =\mathrm{SR} / \mathrm{EF} \\
\mathrm{UCL} & =\mathrm{SR} \times \mathrm{EF}
\end{aligned}
$$

where $\mathrm{EF}=\exp \left(1.96 \sqrt{\left(\mathrm{O}_{\mathrm{i}}\right)}\right)$.

A standardised ratio (SR) of 1 indicates that the area's rate was as expected if the age/gender rates found in the total covered population applied to the PCT/HB area's population structure; a value above 1 indicates that the observed rate was greater than expected given the area's population structure, if the lower confidence limit was above 1 this was statistically significant at the 5\% level. The converse applies to standardised ratios under one.

The combined years analyses are similar to the above except that the observed and expected numbers are summed over the years.

\section{Remaining variability between rates}

Even after standardisation there remains a large amount of variability between PCT/HBs - as can be seen by the large numbers of significantly low or high

Nephron Clin Pract 2012;120(suppl 1):c279-c282 
standard ratios. This is partly because these ratios have only been adjusted for age and gender and have not been adjusted for ethnicity. Much higher rates are expected in populations with a high percentage of patients from South Asian and Black backgrounds. It is hoped that next year we will be able to standardise for ethnicity as well. This data has not been available from ONS until recently.
Caution needed when comparing a PCT/HB's standardised incidence or prevalence ratios over time As the covered areas have changed over time, the 'total' population used for standardisation is different each year. For example, the rate ratios for 2005 and 2006 are not strictly comparable as they are standardised to different populations. However, for most years the change in numbers of covered areas is relatively small. 